\title{
A Hierarchical Approach to Adaptive Local Refinement in Isogeometric Analysis
}

\author{
A.-V. Vuong ${ }^{\mathrm{a}, *}$, C. Giannelli ${ }^{\mathrm{b}}$, B. Jüttler ${ }^{\mathrm{b}}$, B. Simeon ${ }^{\mathrm{c}}$ \\ ${ }^{a}$ Centre for Mathematical Sciences, Technische Universität München, Boltzmannstraße 3, 85748 Garching b. München, Germany \\ ${ }^{b}$ Institute of Applied Geometry, Johannes Kepler University, Altenberger Str. 69, 4040 Linz, Austria \\ ${ }^{c}$ Department of Mathematics, Technische Universität Kaiserslautern, Erwin-Schrödinger-Straße, 67663 Kaiserslautern, Germany
}

\begin{abstract}
Adaptive local refinement is one of the key issues in Isogeometric Analysis. In this article we present an adaptive local refinement technique for Isogeometric Analysis based on extensions of hierarchical B-splines. We investigate the theoretical properties of the spline space to ensure fundamental properties like linear independence and partition of unity. Furthermore, we use concepts well-established in finite element analysis to fully integrate hierarchical spline spaces into the isogeometric setting. This also allows us to access a posteriori error estimation techniques. Numerical results for several different examples are given and they turn out to be very promising.
\end{abstract}

Keywords: Adaptivity, Isogeometric Analysis, Hierarchical B-splines, Local refinement

\section{Introduction}

Adaptive splines which provide local refinement has been studied as an effective tool for surface modeling. Adaptive refinement of spline basis functions has also recently become an active research topic within the framework of isogeometric analysis $[1,2]$, which combines numerical simulation with computer aided design (CAD) geometry by using exact CAD representations like non-uniform rational B-splines (NURBS) into the analysis model. Isogeometric analysis has been applied to a wide range of problems from fluid-structure interaction [3], shape optimization [4], shell analysis [5] to electromagnetics [6], just to name a few. Nevertheless, local refinement is still a key issue due to the tensor-product structure which makes it difficult to obtain finer grids without propagation of the refinement.

Particular attention has been devoted to the application of the promising concept of T-splines [7, 8] into the isogeometric context $[9,10]$. $\mathrm{T}-$ splines allow to break the rigidity of the rectangular topology which characterizes the standard NURBS model, currently used in commercial CAD systems, by considering a mesh — generally indicated as T-mesh — in the parameter domain with axis-aligned edges, where T-junctions (similar to hanging nodes in standard finite elements) are permitted. However, the need to overcome certain limitations of T-splines, in particular with respect to the linear dependence of T-spline blending functions corresponding to particular T-meshes [11] and to the locality of the refinement [9], requires further investigations for the identification of geometric representations suitable for analysis.

\footnotetext{
${ }^{*}$ Corresponding author

Email addresses: vuong@ma.tum.de (A.-V. Vuong), carlotta.giannelli@jku.at (C. Giannelli), bert.juettler@jku.at (B. Jüttler), simeon@mathematik .uni-kl.de (B. Simeon)
}

For this reason, the most recent developments in the field of T-splines include the introduction of "analysis-suitable" Tspline spaces [12]. This restricted set of T-splines allows to guarantee linear independence and restricts the number of additional control points generated by the refinement algorithm to a minimum. These kind of T-spline spaces, however, need a more complicated refinement algorithm, and they still require additional knot insertions beyond the ones specified by the application (by the user and/or by an error estimator).

Other authors consider hierarchical spline spaces over Tmeshes with reduced regularity, such as bicubic $C^{1}$ splines [13, 14]. Once again, these spaces can be considered as special Tsplines [11]. These splines are closely related to classical Hermite elements (e.g. bicubic rectangles) for FEM with hanging nodes, and recently, they were used for isogeometric simulation [15]. The required local behavior of the refinement algorithm and linear independence can be guaranteed easily. The price to pay for this, however, is the reduced regularity of the basis, which increases the number of degrees of freedom needed to obtain the same accuracy.

In order to allow local editing of tensor-product spline surfaces at different levels of detail, Forsey and Bartels [16] introduced hierarchical B-splines as an accumulation of tensorproduct splines with nested knot vectors. More precisely, they modified existing surfaces by locally adding patches representing finer details. Later, Kraft [17] identified a basis for the spaces spanned by these splines and considered their stability. So far, however, these hierarchical splines have found only few applications in geometric design and no applications in isogeometric analysis. The hierarchical model allows complete local control of the refinement by using a spline hierarchy whose levels identify subsequent levels of refinement for the underlying geometric representation.

Working with hierarchies of finite-dimensional subspaces 
offers also a number of advantages from the numerical point of view. For one, there is a straightforward connection to stateof-the-art iterative solvers and preconditioning techniques for large-scale linear systems [18], and furthermore, adaptive refinement and numerical linear algebra may be tightly linked at the algorithmic level [19].

Besides preserving the exact geometry, one of the attractive features of isogeometric analysis is that it offers basis functions with higher smoothness than in standard finite elements [20]. This extra smoothness leads in many cases to better convergence properties [21] and is also valuable when dealing with partial differential equations that demand for $H^{2}$-regularity, such as plate and certain shell problems. Accordingly, for a general local refinement approach it is vital that it can handle and inherit any given degree of continuity. Hierarchical spline spaces are able to meet this requirement. On the other hand, the usage of multiple knots that lead to a decrease of smoothness is also possible.

After this prelude on the motivation for our work, we present in this article the idea of hierarchical B-splines for local adaptive refinement in isogeometric analysis. We rely on a sound theoretical foundation supporting the spline space as well as established finite element concepts and a posteriori error estimation to elaborate this approach. Key properties such as linear independence of the corresponding basis, locality of the refinement process, and arbitrary smoothness can be accomplished in this way, and, moreover, the first computational examples turn out to be promising.

Before starting with the comprehensive exposition we give a short example to illustrate the underlying idea. In Fig. 1, a onedimensional refinement step is shown. At the top, quadratic B-splines are plotted for a given equidistant knot vector. The plot in the middle displays the B-splines after each knot span has been subdivided by knot insertion. The aim now is to locally refine the right half of the original B-splines whereas the left half remains coarse. Obviously, this could also be realized by means of knot insertion, but in multiple dimensions this would result in a propagation of the refinement due to the tensor-product structure. Instead, we replace the coarse basis function which are located at the right (marked by a dashed line) by fine basis function (marked by a solid line), and in this way we create the basis shown at the bottom. The appropriate combination of basis function at different refinement levels is the very simple and powerful idea of hierarchical refinement. The generalization of this process to more complex settings and its properties will be discussed in the following.

The outline of this article is as follows. In Section 2, the hierarchical B-splines are introduced and discussed from a general geometric point of view. Especially it will be shown that favorable properties can be proved and hold for this basis by construction. In Section 3 the hierarchical concept is combined with the framework of isogeometric analysis. To ensure compatibility with finite element routines, the equivalent of an element is introduced and active elements and basis functions at the different levels are defined. An adaptive isogeometric method is then outlined, equipped with standard techniques for error estimation and an extension of the marking algorithm for selecting the regions to be refined. The refinement behavior is furthermore investigated, and it is demonstrated that no undesirable insertion of extra grid points may occur. In Section 4, finally, the hierarchical refinement is applied to several examples while conclusions are drawn in Section 5.

\section{Hierarchical splines}

The only requirement of the philosophy which characterizes the hierarchical approach is a refinable nature of the underlying basis functions defined on nested approximation spaces. Local control of the refinement is achieved through an adaptive procedure that is exclusively based on basis refinement. In this work we consider hierarchical B-spline spaces, but others family of basis functions which exhibit analogous properties and similarly allow adaptive refinement may also be used to define spline hierarchies suitable for analysis.

After introducing some basic notions, in this section we show how to construct a piecewise polynomial non-negative basis composed by locally supported basis functions which can also be modified to form a partition of unity. Moreover, we outline that the hierarchical construction of the spline basis directly implies a nested nature of the corresponding space hierarchy.

\subsection{Tensor product B-spline spaces}

A bivariate tensor-product $\mathrm{B}$-spline space $\mathcal{B}$ is defined by specifying the polynomial degree $(p, q)$ and the horizontal and vertical knots vectors

$$
U=\left\{u_{0} \leq u_{1} \leq \ldots \leq u_{n+p+1}\right\}, \quad V=\left\{v_{0} \leq v_{1} \leq \ldots \leq v_{m+q+1}\right\},
$$

which contain non-decreasing parametric real values so that

$$
0 \leq \mu(U, u) \leq p+1 \quad \text { and } \quad 0 \leq \mu(V, v) \leq q+1
$$

are the multiplicities of the parameter values in the knot vectors (the multiplicity $\mu(X, x)$ is zero if the given value $x$ is not a knot in $X$ ). The space $\mathcal{B}$ is spanned by the tensor-product B-splines

$$
N_{i, j}(u, v)=N_{i, p, U}(u) N_{j, q, V}(v),
$$

where (fractions with zero denominators are considered zero)

$$
\begin{aligned}
& N_{i, 0, U}(u)= \begin{cases}1 & \text { for } u_{i} \leq u<u_{i+1} \\
0 & \text { otherwise }\end{cases} \\
& N_{i, p, U}(u)=\frac{u-u_{i}}{u_{i+p}-u_{i}} N_{i, p-1, U}(u)+\frac{u_{i+p+1}-u}{u_{i+p+1}-u_{i+1}} N_{i+1, p-1, U}(u)
\end{aligned}
$$

for $i=0, \ldots, n$, and the same for $N_{j, q, V}(v)$ replacing $i, p, u$ in (1)-(2) with $j, q, v$ for $j=0, \ldots, m$, are the standard univariate $\mathrm{B}-$ spline basis functions.

Any B-spline function $f(u, v) \in \mathcal{B}$ can be described as

$$
f(u, v)=\sum_{i=0}^{n} \sum_{j=0}^{m} d_{i, j} N_{i, j}(u, v),
$$

with

$$
[u, v] \in\left[u_{p}, u_{n+1}\right] \times\left[v_{q}, v_{m+1}\right],
$$



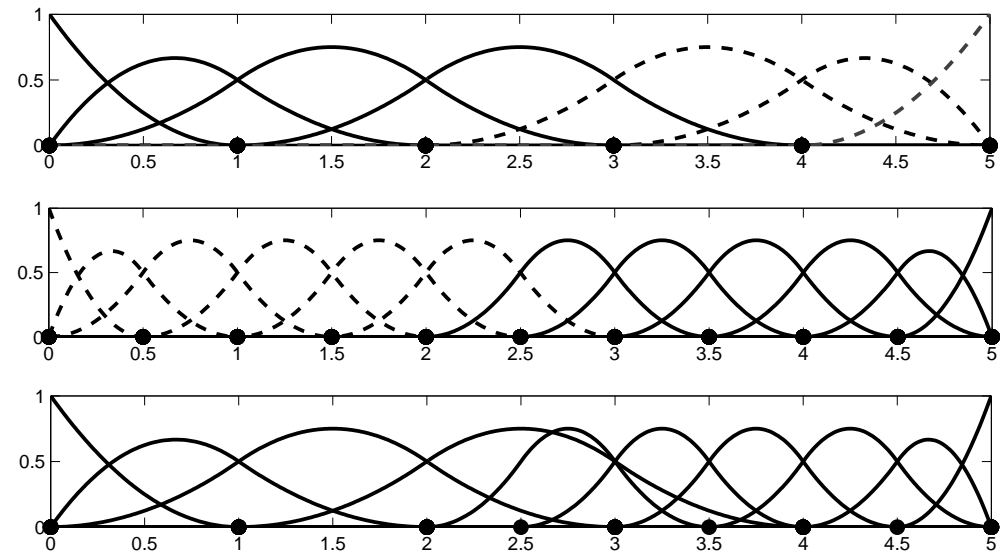

Figure 1: Initial basis (above), refined basis (middle), suitable combination of both (below); selected basis function are marked by a solid line

in terms of the de Boor control points $d_{i, j}$ which form the control net associated to the parametric representation.

The following properties of the space $\mathcal{B}$ and its basis

$$
\mathcal{N}=\left\{N_{i, j}: i=0, \ldots, n, j=0, \ldots, m\right\}
$$

are well known.

(1) Local support:

$$
\left\{(u, v): N_{i, j}(u, v) \neq 0\right\}=\left(u_{i}, u_{i+p+1}\right) \times\left(v_{j}, v_{j+q+1}\right) .
$$

(2) Local linear independence: on any open set $\Omega^{\prime} \subset \Omega$ the B-splines having some support in $\Omega^{\prime}$ are linearly independent on $\Omega^{\prime}$.

(3) Positivity: $N_{i, j}(u, v)>0$ for all $(u, v) \in\left(u_{i}, u_{i+p+1}\right) \times$ $\left(v_{j}, v_{j+q+1}\right)$.

(4) Partition of unity: $\sum_{i=0}^{n} \sum_{j=0}^{m} N_{i, j}(u, v)=1$ for all $(u, v) \in$ $\left[u_{p}, u_{n+1}\right] \times\left[v_{q}, v_{m+1}\right]$.

(5) Smoothness related to knot multiplicity.

The B-spline basis can be generalized to the rational case by associating to each control point $d_{i, j}$ a positive weight $w_{i, j}$ to introduce the projective control points

$$
D_{i, j}=\left(\begin{array}{c}
w_{i, j} d_{i, j} \\
w_{i, j}
\end{array}\right)
$$

which define a projective B-spline function

$$
F(u, v)=\sum_{i=0}^{n} \sum_{j=0}^{m} D_{i, j} N_{i, j}(u, v)
$$

in homogeneous coordinates. By projecting $F(u, v)$ back to affine coordinates we obtain the non-uniform rational B-spline (NURBS)

$$
f(u, v)=\sum_{i=0}^{n} \sum_{j=0}^{m} d_{i, j} R_{i, j}(u, v)
$$

where the NURBS basis functions

$$
R_{i, j}(u, v)=\frac{w_{i, j} N_{i, j}(u, v)}{\sum_{i=0}^{n} \sum_{j=0}^{m} w_{i, j} N_{i, j}(u, v)}
$$

inherit the distinguishing properties which characterize B-spline basis functions.

In order to represent basis functions in the parameter space we can consider different types of anchors, namely

1. naive anchors:

- if both degrees are even, each basis function is identified with the central elementary cell of its support,

- if both degrees are odd, each basis function is identified with the central point (intersection of knot lines) of its support;

- in case of mixed degrees (even/odd), each basis function is identified with the central edge of its support;

2. Greville abscissae: the $(u, v)$ coordinates associated to each basis function $N_{i, j}$ are defined as averages of subsequent knots

$$
\xi_{i}=\frac{1}{p} \sum_{k=1}^{p} u_{i+k}, \quad \eta_{j}=\frac{1}{q} \sum_{k=1}^{q} v_{j+k} .
$$

They satisfy

$$
u=\sum_{i=0}^{n} \xi_{i} N_{i, p, U}(u) \text { and } \quad v=\sum_{j=0}^{m} \eta_{j} N_{j, q, V}(v) .
$$

\subsection{Nested spaces and domains}

We consider a finite sequence of $N$ bivariate B-spline spaces $\left(\mathcal{B}^{\ell}\right)_{\ell=0, \ldots, N-1}$ which are assumed to be nested,

$$
\mathcal{B}^{0} \subset \mathcal{B}^{1} \subset \cdots \subset \mathcal{B}^{N-1}
$$

together with a finite sequence of $N$ bounded open sets $\left(\Omega^{\ell}\right)_{\ell=0, \ldots, N-1}$ with

$$
\Omega^{N-1} \subseteq \Omega^{N-2} \subseteq \cdots \subseteq \Omega^{0}, \quad \Omega^{N}=\emptyset,
$$


which define the nested domains for the spline hierarchy (see Figure 2). Note that this includes the case when $\Omega^{\ell}=\emptyset$ for $\ell>\ell^{0}$. Starting with an initial degree $\left(p^{0}, q^{0}\right)$ characterizing $\mathcal{B}^{0}$, each subsequent spline space $\mathcal{B}^{\ell+1} \supset \mathcal{B}^{\ell}, \ell=0, \ldots, N-2$, is defined by specifying the polynomial degree $\left(p^{\ell+1}, q^{\ell+1}\right)$ with

$$
p^{\ell+1} \geq p^{\ell}, \quad q^{\ell+1} \geq q^{\ell}, \quad \ell=0, \ldots, N-2,
$$

and it is spanned by a tensor-product B-spline basis $\mathcal{N}^{\ell+1}$ defined on the two knot sequences $U^{\ell+1}, V^{\ell+1}$, containing the horizontal and vertical knots, respectively. These knot sequences are also nested, namely

$$
U^{\ell} \subset U^{\ell+1}, \quad V^{\ell} \subset V^{\ell+1}, \quad \ell=0, \ldots, N-2 .
$$

In order to obtain nested spaces, we need to assume that

$\mu\left(U^{\ell+1}, x\right)-\mu\left(U^{\ell}, x\right) \geq p^{\ell+1}-p^{\ell}, \quad \mu\left(V^{\ell+1}, y\right)-\mu\left(V^{\ell}, y\right) \geq q^{\ell+1}-q^{\ell}$,

for all $x, y$ with $\ell=0, \ldots, N-2$. These conditions are necessary and sufficient.

At each level the boundary $\partial \Omega^{\ell}, \ell=0, \ldots, N-1$, may be aligned with the knot lines of $\mathcal{B}^{\ell-1}$ (strong condition) or $\mathcal{B}^{\ell}$ (weak condition). If not specified otherwise, we always assume just the satisfaction of the weak condition on domain boundaries.

\subsection{The hierarchical B-spline basis}

In the remainder of the paper, we consider the support of each function $f$ restricted to the domain $\Omega^{0}$ by defining

$$
\operatorname{supp} f=\left\{(x, y): f(x, y) \neq 0 \wedge(x, y) \in \Omega^{0}\right\} .
$$

By slightly generalizing the selection mechanism for the underlying tensor product $\mathrm{B}-$-spline bases $\mathcal{N}^{0}, \ldots, \mathcal{N}^{N-1}$ introduced in [17], the basis $\mathcal{K}$ for the hierarchical spline space is defined as follows.

Definition 1. The basis $\mathcal{K}$ of the hierarchical spline space is recursively constructed as described below.

(I) Initialization: $K^{0}=\left\{\tau \in \mathcal{N}^{0}: \operatorname{supp} \tau \neq \emptyset\right\}$.

(II) Construction of $K^{\ell+1}$ from $K^{\ell}$ : recursive case.

$$
K^{\ell+1}=K_{A}^{\ell+1} \cup K_{B}^{\ell+1}, \quad \ell=0, \ldots, N-2,
$$

where

$$
K_{A}^{\ell+1}=\left\{\tau \in K^{\ell}: \operatorname{supp} \tau \nsubseteq \Omega^{\ell+1}\right\}
$$

and

$$
K_{B}^{\ell+1}=\left\{\tau \in \mathcal{N}^{\ell+1}: \operatorname{supp} \tau \subseteq \Omega^{\ell+1}\right\} .
$$

(III) $\mathcal{K}=K^{N-1}$.

As shown in Figure 3, in the initialization step we select all basis functions of the underlying $\mathrm{B}$-spline basis $\mathcal{N}^{0}$ whose support overlaps $\Omega^{0}$, while in the recursive step

- first, considering $K_{A}^{\ell+1}$, we add all basis functions $\tau$ of the previous level whose support is not entirely contained in $\Omega^{\ell+1}$,

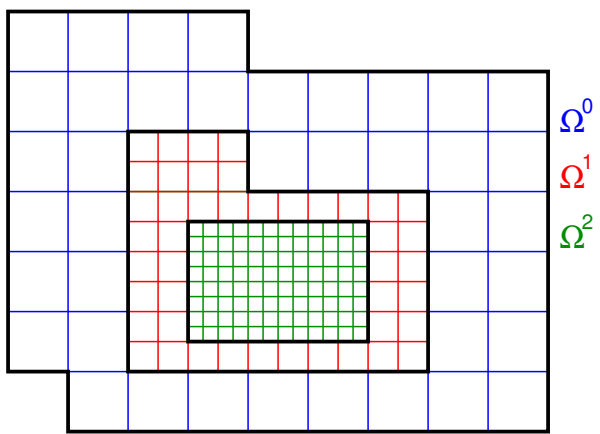

(a) Strong conditions on domain boundaries.

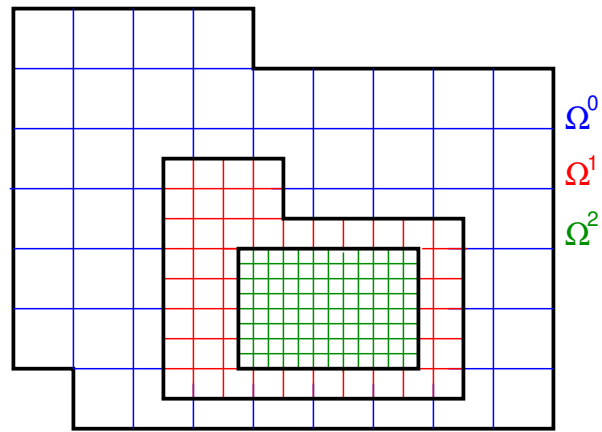

(b) Weak conditions on domain boundaries.

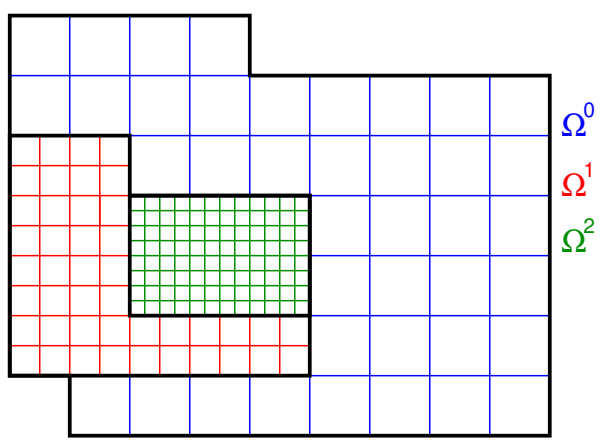

(c) The case $\partial \Omega^{\ell} \cap \partial \Omega^{\ell+1} \neq \emptyset, \ell=0,1$.

Figure 2: Nested domains for the spline hierarchy.

- then, $\Omega^{\ell+1}$ is covered by the refined basis functions in $\mathcal{N}^{\ell+1}$ contained in $K_{B}^{\ell+1}$.

The situations not covered in [17] (weak condition on domain boundaries, partly overlapping domain boundaries) are shown in Figure 2 (b)-(c). Some examples for this selection of basis functions are shown in Figure 4.

The linear independence of the hierarchical basis functions follows immediately from Definition 1.

Lemma 2. The functions in $\mathcal{K}$ are linearly independent.

Proof. The sum

$$
0=\sum_{\tau \in \mathcal{K}} d_{\tau} \tau
$$

can be re-arranged as

$$
0=\sum_{\tau \in \mathcal{K} \cap \mathcal{N}^{0}} d_{\tau} \tau+\sum_{\tau \in{\mathcal{K} \cap \mathcal{N}^{1}}} d_{\tau} \tau+\ldots+\sum_{\tau \in \mathcal{K}^{\prime} \cap \mathcal{N}^{N-1}} d_{\tau} \tau
$$




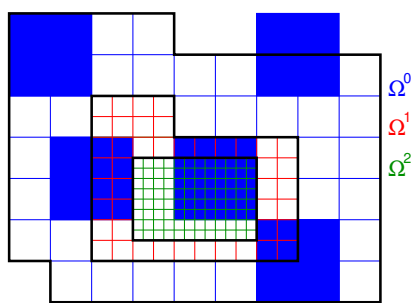

(a) Step 0

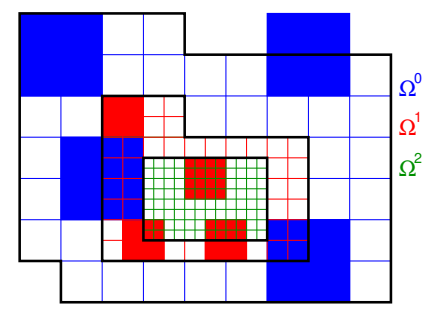

(b) Step 1

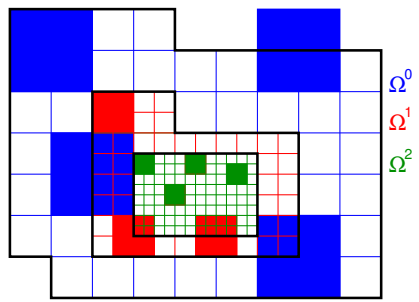

(c) Step 2

Figure 3: Selection of basis functions by the iterative procedure described in Definition 1 : bilinear case $\left(p^{\ell}, q^{\ell}\right)=(1,1), \ell=0,1,2$. Sampled basis functions are represented by their supports.

Since the functions in $\mathcal{K} \cap \mathcal{N}^{0}$ are a subset of $\mathcal{N}^{0}$, they are linearly independent. Only these functions are non-zero on $\Omega^{0} \backslash \Omega^{1}$, hence, in view of their local linear independence, we conclude that $d_{\tau}=0$ for $\tau \in \mathcal{K} \cap \mathcal{N}^{0}$. Analogously, when we consider all next sums in the sequence, we may observe that for each $\ell=1, \ldots, N-1$ the functions in $\mathcal{K} \cap \mathcal{N}^{\ell}$ are linearly independent. Except for functions already considered in the previous sums, namely in $\mathcal{K} \cap \mathcal{N}^{0}, \ldots, \mathcal{K} \cap \mathcal{N}^{\ell-1}$, only the functions in $\mathcal{K} \cap \mathcal{N}^{\ell}$ are non-zero on $\Omega^{\ell} \backslash \Omega^{\ell+1}$. This implies that $d_{\tau}=0$ for $\tau \in \mathcal{K} \cap \mathcal{N}^{\ell}$ with $\ell=1, \ldots, N-1$.

The following Lemma proves the nested nature of the spaces spanned by the sequence of spline bases $K^{0}, \ldots, K^{N-1}$ which appear in the different levels of the hierarchy.

Lemma 3. Let $K^{0}, \ldots, K^{N-1}$ be the spline bases considered by the iterative procedure of Definition 1 . We have

$$
\operatorname{span} K^{\ell} \subseteq \operatorname{span} K^{\ell+1}, \quad \ell=0, \ldots, N-2 .
$$

Proof. Any function $f \in \operatorname{span} K^{\ell}$ with $\ell=0, \ldots, N-2$, can be expressed as

$$
f=\sum_{\tau \in K^{\ell}} d_{\tau} \tau=\sum_{\tau \in K^{\ell}, \operatorname{supp} \tau \nsubseteq \Omega^{\ell+1}} d_{\tau} \tau+\sum_{\tau \in K^{\ell}, \operatorname{supp} \tau \subseteq \Omega^{\ell+1}} d_{\tau} \tau .
$$

The first sum in the right-hand side of the above relation collects all basis functions in $K_{A}^{\ell+1}$. In view of the nested nature of the underlying spaces $\mathcal{B}^{0}, \ldots, \mathcal{B}^{N-1}$, we can express each basis function $\tau \in \mathcal{N}^{\ell}$ as linear combination of basis functions which belong to $\mathcal{N}^{\ell+1}$, namely

$$
\tau=\sum_{\sigma \in \mathcal{N}^{\ell+1}, \operatorname{supp} \sigma \subseteq \operatorname{supp} \tau} c_{\sigma}^{\ell+1}(\tau) \sigma,
$$

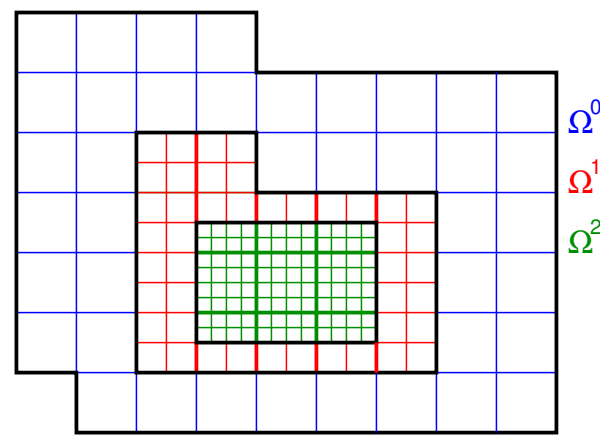

(a) The case of different degrees $\left(p^{0}, q^{0}\right)=(1,1)$, $\left(p^{1}, q^{1}\right)=(2,1),\left(p^{2}, q^{2}\right)=(2,2)$. Double knot lines are shown in bold.

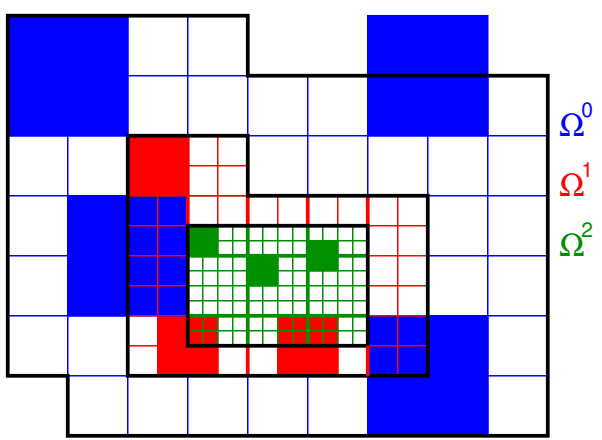

(b) The case of different degrees $\left(p^{0}, q^{0}\right)=(1,1)$, $\left(p^{1}, q^{1}\right)=(2,1),\left(p^{2}, q^{2}\right)=(2,2)$.

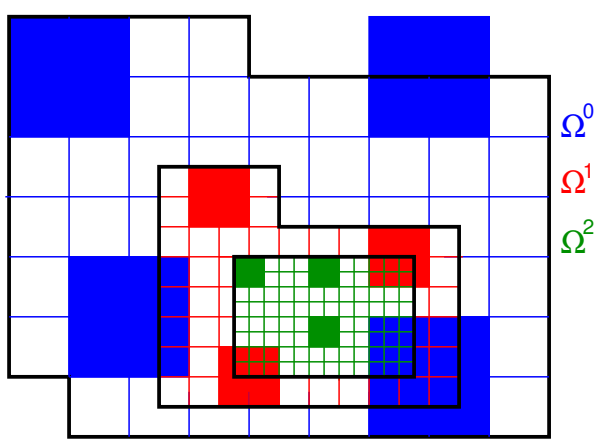

(c) The case of weak condition on domain boundaries (same degrees of Figure 3).

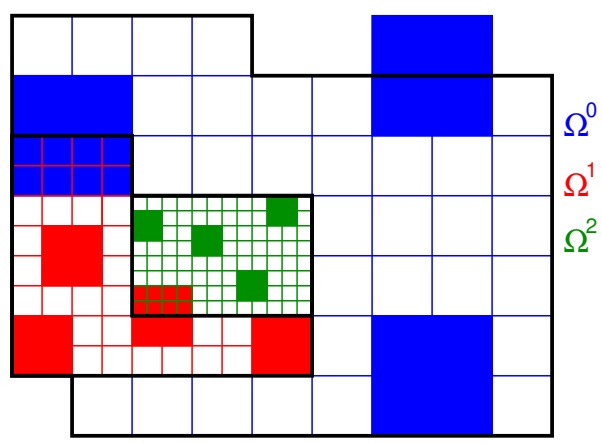

(d) The case $\partial \Omega^{\ell} \cap \partial \Omega^{\ell} \neq \emptyset, \ell=0,1$ (same degrees of Figure 3).

Figure 4: Selection of basis functions: sampled basis functions are represented by their supports. 
where we denote by $c_{\sigma}^{\ell+1}(\tau)$ the coefficient of $\sigma$ in this expansion of $\tau$. By substituting the above relation for $\tau$ into the second sum of the right-hand side of (5), we obtain

$$
f=\sum_{\tau \in K_{A}^{\ell+1}} d_{\tau} \tau+\sum_{\tau \in K^{\ell}, \operatorname{supp} \tau \subseteq \Omega^{\ell+1}} d_{\tau}\left(\sum_{\sigma \in \mathcal{N}^{\ell+1}, \operatorname{supp} \sigma \subseteq \operatorname{supp} \tau} c_{\sigma}^{\ell+1}(\tau) \sigma\right)
$$

Since $\operatorname{supp} \sigma \subseteq \operatorname{supp} \tau \subseteq \Omega^{\ell+1}$ and observing that additional coefficients of basis functions $\sigma$ whose support is not contained in the support of the function $\tau$ considered in (6) are zero, we may consider supp $\sigma \subseteq \Omega^{\ell+1}$, and swapping the order of the two rightmost sums on the above equation leads to

$$
\begin{aligned}
f & =\sum_{\tau \in K_{A}^{\ell+1}} d_{\tau} \tau+\sum_{\sigma \in \mathcal{N}^{\ell+1}, \operatorname{supp} \sigma \subseteq \Omega^{\ell+1}}\left(\sum_{\tau \in K^{\ell}, \operatorname{supp} \tau \subseteq \Omega^{\ell+1}} d_{\tau} c_{\sigma}^{\ell+1}(\tau)\right) \sigma \\
& =\sum_{\tau \in K_{A}^{\ell+1}} d_{\tau} \tau+\sum_{\sigma \in K_{B}^{\ell+1}} d_{\sigma} \sigma
\end{aligned}
$$

where

$$
d_{\sigma}=\sum_{\tau \in K^{\ell}, \operatorname{supp} \tau \subseteq \Omega^{\ell+1}} d_{\tau} c_{\sigma}^{\ell+1}(\tau) .
$$

The first sum on the last row of (7) belongs to the span of $K_{A}^{\ell+1}$, while the second sum therein belongs to the span of $K_{B}^{\ell+1}$. Hence, $f \in \operatorname{span} K^{\ell+1}$.

Since the hierarchical basis functions are contained in the coarsest level of the spline hierarchy, we may define Greville abscissae with respect to the basis $\mathcal{K}$ by the identity

$$
\left(\begin{array}{l}
u \\
v
\end{array}\right)=\sum_{\tau \in \mathcal{K}}\left(\begin{array}{l}
\xi(\tau) \\
\eta(\tau)
\end{array}\right) \tau
$$

Note that these Greville abscissae with respect to $\mathcal{K}$ are generally different from the ones defined in (4).

\subsection{Enlarging the subdomains of spline hierarchies}

As it will be described in more details in the next section, mesh refinement based on a posteriori error estimators is a key ingredient for developing an effective isogeometric solver. This is the main motivation for investigating possible extensions of tensor product splines which provide an adaptive local control of the refinement, together with their suitable application into the analysis phase. Starting with an initial mesh grid, the error estimator iteratively identifies areas of the mesh which require to be locally refined. Typically, a local refinement proceeds as follows: we start with a uniform discretization, i.e., $\Omega^{1} \supseteq$ $\ldots \supseteq \Omega^{N-1}=\emptyset$. The error estimator indicates regions to be refined, then $\Omega^{1}$ is increased. In each step, one may expect that the maximum refinement level (the biggest $\ell$ so that $\Omega^{\ell} \neq$ $\emptyset)$ increases by one. It may happen, however, that we need to refine areas of the domain which are not completely contained in the subdomain which corresponds to the current maximum level of refinement. We can then consider another sequence of "enlarged" nested domains $\left\{\hat{\Omega}^{\ell}\right\}_{\ell=0, \ldots, N-1}$ which allows to refine in the additional regions as indicated by the error estimator (see Figure 5).

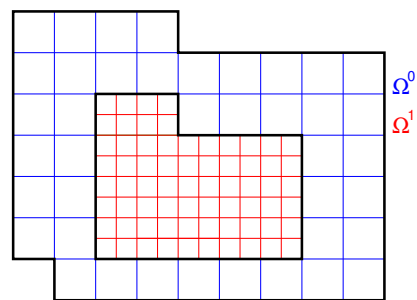

(a) Original nested domains.

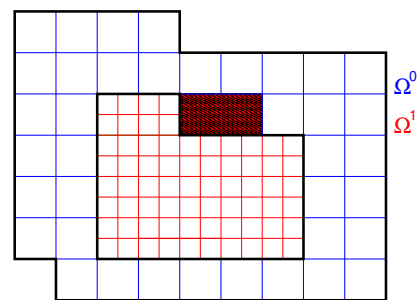

(b) Area selected to be refined.

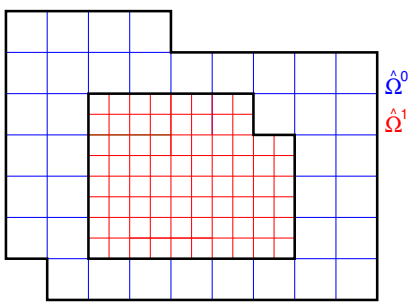

(c) Enlarged nested domains.

Figure 5: Nested enlargment of subdomains for the spline hierarchy.

The following proposition ensures that the nested nature of the spline spaces is still preserved,

Proposition 4. Consider the two sequence of nested domains

$$
\Omega^{N-1} \subseteq \Omega^{N-2} \subseteq \ldots \subseteq \Omega^{0} \quad \text { and } \quad \hat{\Omega}^{N-1} \subseteq \hat{\Omega}^{N-2} \subseteq \ldots \subseteq \hat{\Omega}^{0},
$$

together with the corresponding hierarchical bases $K^{\ell}$ and $\hat{K}^{\ell}$, constructed according to Definition 1 , for $\ell=0, \ldots, N-1$. If $\Omega^{\ell} \subseteq \hat{\Omega}^{\ell}$, for $\ell=0, \ldots, N-1$, then

$$
\operatorname{span} K^{\ell} \subseteq \operatorname{span} \hat{K}^{\ell}
$$

Proof. Since at each level $\ell$, the sequence of nested domains $\left\{\hat{\Omega}^{\ell}\right\}_{\ell=0, \ldots, N-1}$, enlarged with respect to the sequence $\left\{\Omega^{\ell}\right\}_{\ell=0, \ldots, N-1}$, covers a wider area of the domain by refined function of the next basis in the underlying sequence $\left\{\mathcal{N}^{\ell}\right\}_{\ell=0, \ldots, N-1}$, if $K^{\ell}=K_{A}^{\ell} \cup K_{B}^{\ell}$ and $\hat{K}^{\ell}=\hat{K}_{A}^{\ell} \cup \hat{K}_{B}^{\ell}$, we have

$$
K_{A}^{\ell} \supseteq \hat{K}_{A}^{\ell}, \quad K_{B}^{\ell} \subseteq \hat{K}_{B}^{\ell},
$$

and $\operatorname{span} K_{A}^{\ell} \backslash \hat{K}_{A}^{\ell} \subseteq \operatorname{span} K_{B}^{\ell}$. Hence, the space spanned by the basis $K^{\ell}$ is contained in the space spanned by $\hat{K}^{\ell}$.

\subsection{A weighted hierarchical basis}

The key property of Bernstein/B-spline representations is that the related curve or surface is a convex combination of the underlying control points. In order to achieve this within the framework of the hierarchical approach, we may modify $\mathcal{K}$ to obtain a hierarchical basis $\mathcal{W}$ which, at each level, forms a partition of unity. Since affine transformations leave invariant barycentric combinations, we will regain the invariance by affine transformation property. Moreover, since B-spline functions are non-negative, by defining hierarchical spline basis functions which also form a partition of unity, the curve/surface will be a convex combination of its control points, implying the convex hull property. 
In view of Lemma 3, if the constant function 1 belongs to the span of $K^{0}$ we can always represent it by the hierarchical basis $\mathcal{K}$ as

$$
1=\sum_{\tau \in \mathcal{K}} w_{\tau} \tau
$$

Hence, if for each basis function $\tau$ in $\mathcal{K}$ we define a related weighted basis function $\omega=w_{\tau} \tau$, assuming $w_{\tau} \neq 0$ we obtain the normalized hierarchical basis

$$
\mathcal{W}=\left\{\omega=w_{\tau} \tau: \tau \in \mathcal{K} \wedge 1=\sum_{\tau \in \mathcal{K}} w_{\tau} \tau\right\}
$$

which forms a partition of unity, namely

$$
\sum_{\omega \in \mathcal{W}} \omega=1
$$

Lemma 5. Any weight $w_{\tau}$ associated to a function $\tau \in \mathcal{K}$ by means of equation (8) is greater or equal to zero.

Proof. Since $1 \in \operatorname{span} K^{0}$ we can write

$$
1=\sum_{\gamma \in \mathcal{N}^{0}} d_{\gamma} \gamma
$$

with each $d_{\gamma} \geq 0$, and then

$$
1=\sum_{\gamma \in \mathcal{N}^{0}, \operatorname{supp} \gamma \nsubseteq \Omega^{1}} d_{\gamma} \gamma+\sum_{\gamma \in \mathcal{N}^{0}, \operatorname{supp} \gamma \subseteq \Omega^{1}} d_{\gamma} \gamma .
$$

The first sum in the right hand-side of the above relation collects all basis functions in $K_{A}^{1}$. As we already argued in the proof of the previous Lemma, we can express each basis function $\tau \in \mathcal{N}^{0}$ as a linear combination of basis functions which belong to $\mathcal{N}^{1}$, namely

$$
\gamma=\sum_{\sigma \in \mathcal{N}^{1}} c_{\sigma}^{1}(\gamma) \sigma
$$

with $c_{\sigma}(\gamma) \geq 0$. By substituting the above relation for $\tau$ in the second sum of the right hand-side of (9), we obtain

$$
1=\sum_{\gamma \in K_{A}^{1}} d_{\gamma} \gamma+\sum_{\gamma \in \mathcal{N}^{0}, \operatorname{supp} \gamma \subseteq \Omega^{1}} d_{\gamma}\left(\sum_{\sigma \in \mathcal{N}^{1}, \operatorname{supp} \sigma \subseteq \Omega^{1}} c_{\sigma}^{1}(\gamma) \sigma\right) .
$$

Swapping the order of the two rightmost sums leads to

$$
\begin{aligned}
1 & =\sum_{\gamma \in K_{A}^{1}} d_{\gamma} \gamma+\sum_{\sigma \in \mathcal{N}^{1}, \operatorname{supp} \sigma \subseteq \Omega^{1}}\left(\sum_{\gamma \in \mathcal{N}^{0}, \operatorname{supp} \gamma \subseteq \Omega^{1}} d_{\gamma} c_{\sigma}^{1}(\gamma)\right) \sigma \\
& =\sum_{\tau \in K_{A}^{1}} d_{\tau} \tau+\sum_{\sigma \in K_{B}^{1}} d_{\sigma} \sigma,
\end{aligned}
$$

where

$$
d_{\sigma}=\sum_{\gamma \in \mathcal{N}^{0}, \operatorname{supp} \gamma \subseteq \Omega^{1}} d_{\gamma} c_{\sigma}^{1}(\gamma) \geq 0 .
$$

The first sum on the second row of (10) belongs to the span of $K_{A}^{1}$, while the second sum therein belongs to the span of $K_{B}^{1}$ and the involved coefficients are all $\geq 0$. We may iterate this procedure visiting each level of the hierarchy and representing each time the constant function 1 as a linear combination of basis functions which belong to the next level in terms of coefficients $\geq 0$. After visiting all levels we obtain equation (8) with each $\omega_{\tau} \geq 0$.

Unfortunately, for some configuration of the hierarchical domain, some of the weights associated to basis functions in the refined level are zero. For instance, this happens when, moving from level $\ell$ to level $\ell+1$ in the hierarchy, there exists no basis function $\tau \in K^{\ell}$ with a support contained in $\Omega^{\ell+1}$, i.e. the refined domain is smaller than the support of basis functions defined on the grid of the previous level. If this is the case, all basis functions in $K^{\ell}$ will be kept in the hierarchical basis and, since we already achieved the partition of unity for the level $\ell$, the weight of any basis function in $\mathcal{N}^{\ell+1}$ whose support is contained in $\Omega^{\ell+1}$ will be zero. The local action of these refined functions will then be canceled. More precisely, this situation appears if and only if there exists a basis function $\tau \in K_{B}^{\ell+1}$ whose support is not contained in the support of a function in $K^{\ell} \backslash K_{A}^{\ell+1}$. This situation may be avoided if we assume the strong condition on domain boundaries and $\Omega^{\ell+1}$ defined as union of supports of basis functions $\tau \in \mathcal{N}^{\ell}$,

$$
\Omega^{\ell+1}=\longdiv { \bigcup _ { \tau \in S } \operatorname { s u p p } \tau },
$$

where $S \subseteq \mathcal{N}^{\ell}$.

The overlap of basis supports can be significantly reduced by using another basis, called truncated basis, which will be described elsewhere. This basis may increase the sparsity of stiffness matrix in isogeometric methods.

\section{Adaptive hierarchical refinement}

In this section we combine the hierarchical approach to local refinement with the method of isogeometric analysis. Aiming at a concise framework, we restrict the discussion to a particular case of the spline spaces introduced in the previous section. Let the following assumptions hold.

A1 The parameter domain is a rectangular domain or without loss of generality the unit square,

$$
\Omega^{0}=[0,1]^{2} .
$$

A2 The boundaries of a refined region are always aligned with the knot lines of $\mathcal{B}^{\ell-1}$, which was referred to as "strong condition" in the previous section.

A3 The degree $(p, q)$ does not change during hierarchical refinement, i.e., we only consider local $h$-refinement.

Nevertheless, this method can be generalized to arbitrary parameter domains with axis-aligned boundaries, and consequently to all geometries that can be represented by parameter domain of this type.

After describing shortly the basics of isogeometric analysis, we interpret next the concept of hierarchical spline spaces from the viewpoint of numerical analysis and use it then as local refinement technique. 


\subsection{Isogeometric analysis}

The starting point for the application of isogeometric analysis and general finite element methods to elliptic problems is the variational formulation of a partial differential equation: find $\varphi \in \mathcal{V}$ such that

$$
a(\varphi, \psi)=\langle l, \psi\rangle \text { for all } \psi \in \mathcal{V}
$$

with coercive bilinear form $a(\cdot, \cdot)$ and test functions $\psi$ in the space $\mathcal{V}:=\left\{\psi \in H^{1}(\Omega), \psi=0\right.$ on $\left.\Gamma_{D}\right\}$ where $\Gamma_{D}$ denotes the Dirichlet boundary. We suppose that the physical domain $\Omega$ is parametrized by a global geometry function

$$
F: \Omega^{0} \rightarrow \Omega, \quad F(u, v)=\sum_{i=0}^{n} \sum_{j=0}^{m} R_{i j}(u, v) d_{i j}
$$

based on NURBS $R_{i j}(u, v)$ defined in (3) and control points $d_{i j}$. The parametrization $F$ is crucial in the following because its properties, in particular the smoothness, are passed to the numerical solution.

The basic idea of isogeometric analysis is to formulate the Galerkin projection with respect to the same basis functions that describe the geometry mapping $F$ in (14). Defined on the parameter domain $\Omega^{0}$, these functions are mapped to the physical domain $\Omega$ by means of $F$ as global push-forward operator. In other words,

$$
\mathcal{V}_{h}=\operatorname{span}\left\{R_{i j} \circ F^{-1}\right\}_{i=0 \ldots n, j=0 \ldots m}
$$

is the finite dimensional subspace for the projection.

\subsection{Local hierarchical refinement}

The idea of hierarchical refinement in isogeometric analysis is simply to replace the standard NURBS basis in (15) by a hierarchical one. The hierarchical basis of Definition 1 is defined on $\Omega^{0}$, possesses favorable properties by construction and is thus a promising candidate for our objective. Note that for the present implementation we do not consider the most general case but only sequences of spline spaces with the assumptions A1-A3 stated above. Further approaches are possible within the framework of hierarchical splines but out of the scope of this paper.

To this end, we proceed in several steps, starting with the concept of an element. So far, the spline functions have been mainly described in terms of their support. In order to access them in a more FEM-like manner, however, we characterize these functions through elements. It has become standard in isogeometric analysis to identify the computational mesh with Cartesian products of knot spans [21]. We adopt this here and call

$$
\left.T_{i j}=\right] u_{i}, u_{i+1}[\times] v_{j}, v_{j+1}[,
$$

with $0 \leq i \leq n+p$ and $0 \leq j \leq m+q$ an element. Note that we include empty knot spans in this definition. These occur for instance due to multiple knots at the boundary of $\Omega^{0}$.

The isogeometric mesh is now defined as the set of all elements,

$$
Q:=\left\{T_{i, j}\right\}_{i=0 \ldots n+p, j=0 \ldots m+q}
$$

An important ingredient of our approach is using a hierarchy of meshes that are constructed consecutively. More specifically, we create a sequence of meshes

$$
Q^{\ell}=\left\{T_{i, j}^{\ell}\right\}_{i=0 \ldots n^{\ell}+p, j=0 \ldots m^{\ell}+q}
$$

with index $\ell=0, \ldots, N-1$ by successive global uniform $h$ refinement, e.g., by inserting a knot in the middle of each nonempty knot span. An example for such a sequence is shown in Fig. 6 and will be used as illustration from now on.
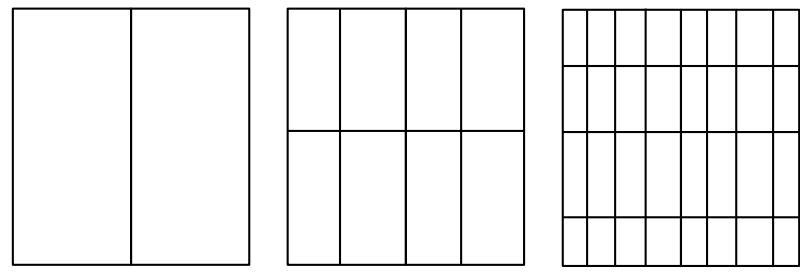

Figure 6: Sequence of h-refined parameter spacto appeares $Q^{\ell}$

The alert reader will notice that the mesh sequence (18) corresponds to the levels of the spline spaces $\mathcal{B}^{\ell}$, and over each isogeometric mesh $Q^{\ell}$ a set of basis functions $\mathcal{N}^{\ell}$ is defined. Due to the underlying $h$-refinement process, this procedure can be also performed for NURBS instead of B-splines.

The higher smoothness offered by isogeometric analysis implies that the support of the basis functions is usually larger than in standard finite elements. For this reason, the connection between the mesh and the basis functions is more subtle and requires particular attention. Even more, when considering the mesh levels and basis functions in the hierarchical approach it is crucial to find an approach that easily provides access to the involved elements and functions across all levels. Only these are considered in the assembly routines for the computation of the system matrices and are therefore called "active" in the following.

\subsubsection{Active elements}

Having multiple levels of knot spans or elements as a starting point it is a key issue to manage these. For each level $\ell$ we select certain elements of $Q^{\ell}$ to be active. More specifically, we call a selection $\mathcal{A}=\bigcup_{\ell=0}^{N-1} A^{\ell}$ of elements with $A^{\ell} \subset Q^{\ell}$ active if they fulfill the following properties.

1. Each element does not have zero area.

2. Two arbitrary elements are disjoint,

$$
\forall T_{1}, T_{2} \in \mathcal{A}: T_{1} \cap T_{2}=\emptyset .
$$

3. The union of all elements forms the whole parametric domain,

$$
\bigcup_{T \in \mathcal{A}} \bar{T}=\Omega^{0}
$$

4. Support condition: For an active element $\hat{T} \in \mathcal{A}$ with level $k>0$, i.e., $\hat{T} \in A^{k}$, there exists a set of elements

$$
M \subseteq \bigcup_{\ell=k}^{N-1} A^{\ell}
$$


which contains the first element itself,

$$
\hat{T} \in M,
$$

and its closure equals the closure of a $(p+1) \times(q+1)$ block of subsequent elements of the lower level $k-1$

$$
\overline{\bigcup_{C \in M} C}=\overline{\bigcup_{r, s=0}^{p} T_{i+r, j+s}^{k-1}}
$$

with indices $i$ and $j$ only dependent on $\hat{T}$.

The first three properties are quite straightforward and ensure a partitioning of the computational domain into non-empty elements. An example is shown in Fig. 7 where eight elements are selected to be active across three levels, starting with the mesh sequence of Fig. 6.
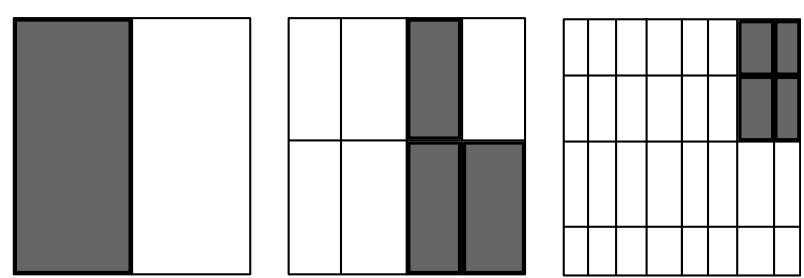

Figure 7: Sequence of $h$-refined parameter spaces with active elements marked in grey

The fourth property needs some further explanation. It only affects elements that are not part of the lowest level. In particular, a uniform grid, where $\mathcal{A} \subset Q^{\ell}$, is an active selection of elements as well. So uniform refinement is still feasible within this framework. For an element from level $k>0$, the set $M$ can be viewed as the minimal refinement region that has to be filled out with elements of level $k$ or higher. In order to replace a coarse basis function from the previous level $(k-1)$, the minimal refinement region $M$ has to be as large as the support of the function, which is the block of $(p+1) \times(q+1)$ elements of level $(k-1)$. This ensures that grid refinement takes effect on the basis functions. Otherwise, it would be impossible to replace the coarse function by finer ones. If Fig. 8, two examples show how this condition affects the refinement region. For the meshes on the left one cannot find a set $S$ that satisfies condition (23) for the highlighted element, whereas in the meshes on the right, the set $M$ is marked in grey. We can identify the sequence of nested domains by

$$
\overline{\Omega^{k}}=\overline{\bigcup_{\ell=k}^{N-1} \bigcup_{C \in A^{\ell}} C .}
$$

The fourth condition is equivalent to (11) and guarantees that a weighted basis $\mathcal{W}$ forming a partition of unity exists.

We note that the support condition only incorporates the degree of the spline functions and, although it may seem complicated, it can be established naturally in combination with an error estimator, see paragraph 3.4 below.

In our hierarchical approach, the active elements serve as analogue of a finite element mesh and form a locally refined
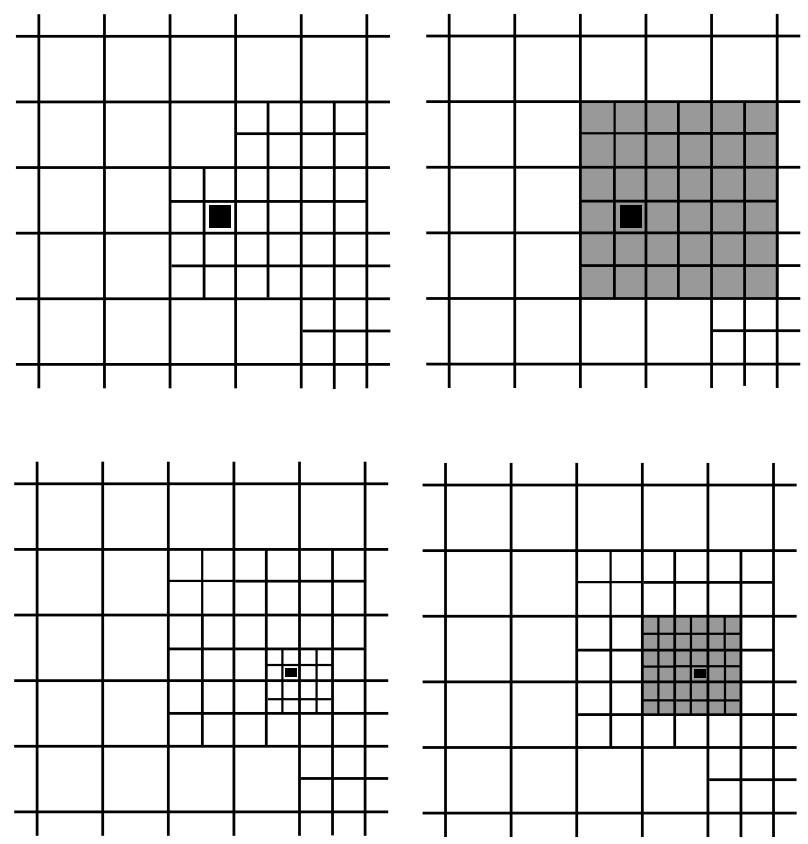

Figure 8: Two examples with degree $p=q=2$ : Violation of the support condition for the selected element marked by a black box (left), altered grid which fulfills the support condition with $M$ shown in grey (right)

structure. Typical operations like quadrature and error estimation are carried out element by element.

\subsubsection{Active basis functions}

After having defined the active elements in the hierarchical structure we can now address the basis functions. Analogously to the element level above, one has to specify an appropriate selection that we call the active basis functions.

The set of active basis functions $\mathcal{K}$ is defined as follows: a function $\beta \in \mathcal{N}^{k}$ of level $k$ is element of $\mathcal{K}$ if

$$
\operatorname{supp} \beta \subset \overline{\Omega^{k}}
$$

and

$$
\operatorname{supp} \beta \nsubseteq \overline{\Omega^{k+1}}
$$

An example is given in Fig. 9, which is derived from Fig. 7 and extended by circles to mark the Greville abscissae. We deliberately do not visualize here the basis functions by means of their support as in Section 2 since we are mainly interested in their overall distribution.

The active functions coincide with the definition of the hierarchical basis and therefore it is justified that we use the same identifier $\mathcal{K}$. Furthermore, the properties proved in Section 2 hold as well: the active basis functions are linearly independent, Lemma 2, and the original space is a subset of the refined space defined by the active basis functions,

$$
\mathcal{B}^{0} \subseteq \operatorname{span} \mathcal{K},
$$

according to Lemma 3. This implies that it is still possible to represent the geometry exactly in the refined space. Furthermore, by scaling as discussed in Section 2.5 the partition of 


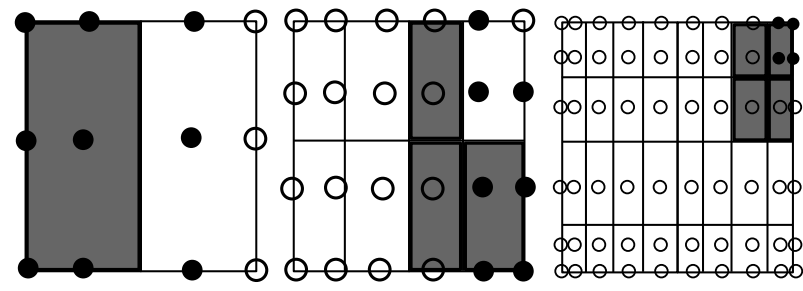

Figure 9: Active functions (black circles), non active functions (white circles) for degree $p=q=2$ based on active element (grey elements)

unity property can be preserved. Summarizing, all the essential properties needed for using the basis functions in isogeometric analysis are ensured by construction.

\subsubsection{Refinement procedure}

The concepts of active elements and active basis functions put us in a position to develop a refinement procedure. Starting from a normal standard tensor-product surface defined in the space $\mathcal{B}^{0}$, the elements to be refined are replaced by elements of a higher level. More specifically, as we employ uniform $h$ refinement a single element is subdivided into four smaller elements, Fig. 10. The underlying spline spaces $\mathcal{B}^{\ell}$ therefore are created by inserting knots that splits each non-empty knot interval into halves.

Again, the small element can be refined as well, so that we get a refined grid over several levels. If we continue to subdivide other active elements, Proposition 4 ensures that this procedure always enlarges the current space and we get a sequence of nested spaces.

In order to simplify the graphical representation of the hierarchies, we visualize here the refinement by showing all active elements within the same domain for degrees $p=q=1$ and $p=q=2$. Again, we use the configuration of Fig. 9 and represent the active basis functions by Greville abscissae. The same approach will be taken to visualize the refinement process for the computational examples in Section 4.

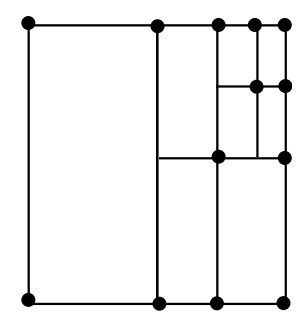

(a) degree $p=q=1$

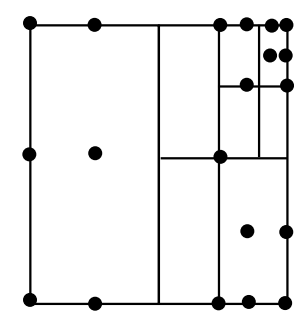

(b) degree $p=q=2$
Figure 10: Refined grid with Greville abscissae of the active functions

Note that the elements of a certain level do not necessarily have the same size or the same ratio of edge lengths. The regular size in the figures was chosen only for the sake of simplicity and comprehensibility.

In the refinement process, the support condition (23) must be taken into account so that the marked region is large enough.
This is the only point in our approach that needs extra attention, and how to take care of it is discussed in the context of error estimation, see below. The selection of elements at different levels created in this way contains all the information that we need to compute the corresponding basis functions. Built upon an initial arbitrary spline space this refinement can be applied to any degree and any continuity.

\subsection{Refinement behavior}

From the discussion so far it is not obvious how the refinement actually behaves, in particular in view of the problems T-splines may face when dealing with refinement along a diagonal. Global refinement is performed by means of knot insertion and therefore follows the direction of the knot lines. From an adaptive refinement procedure we would expect that it also can handle regions that are not aligned with knot lines.

As an example we look at a square domain $[0,1]^{2}$ with the identity as its parametrization and aim at refining the diagonal for degree $p=q=2$. After three successive $h$-refinements in every parameter direction we obtain 64 elements in total in the starting grid. Next, the elements along the diagonal are marked for refinement, taking the support condition (23) into account. I.e., we refine $(p+1) \times(q+1)$-blocks into smaller ones. In Fig. 11, the resulting grid is displayed. It can be seen that a

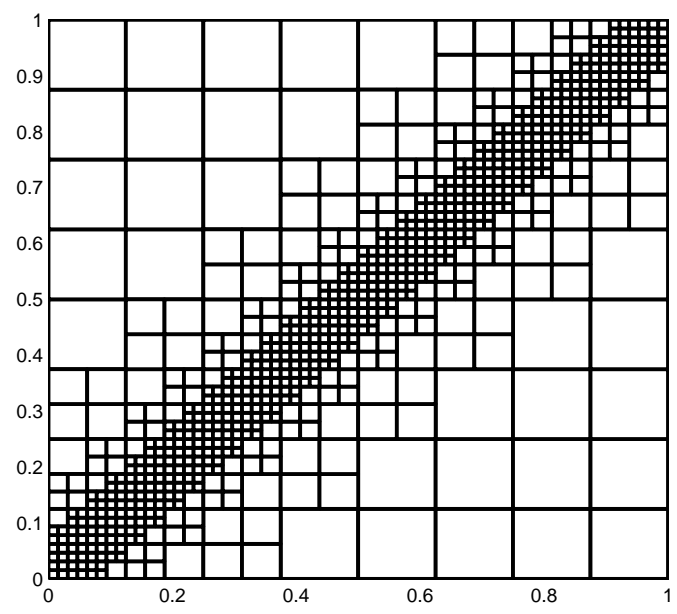

Figure 11: Refinement along the diagonal for degree 2

high resolution at the diagonal can be achieved. The additional refinement beyond the elements along the diagonal is due to the fact that we enforce the support condition during the refinement. Obviously this is a spatially limited effect because it only affects the support of changed basis functions, and accordingly, the refinement will not propagate away from the region of interest and is strictly bounded by a small offset of the diagonal. Some approaches to local refinement decrease the continuity, typically by insertion of multiple knots, to limit the propagation of the refinement. In our case of hierarchical refinement this is not necessary and the discretization preserves the initial degree of continuity.

Another insightful test example in this context is the advectiondiffusion problem discussed in Section 4.3. 


\subsection{Error estimation}

So far, we have concentrated on the refinement procedure, but it is also necessary to discuss the issue of error estimation. The desired quantity, the error

$$
e=\varphi-\varphi_{h}
$$

with exact solution $\varphi$ and numerical approximation $\varphi_{h} \in \mathcal{V}_{h}$, is typically not available and therefore has to be approximated. Furthermore, it is important to spatially localize the estimated error $\eta$ and therefore the estimate is typically computed elementwise

$$
\eta(T) \approx\left\|\left.\left(\varphi-\varphi_{h}\right)\right|_{T}\right\|
$$

in some norm, e.g., in the energy norm. Then according to a marking threshold, e.g.,

$$
\theta=\alpha \max _{k}\left(\eta\left(T_{k}\right)\right)
$$

with $\alpha \in[0,1]$, the elements which are to be refined are marked.

We will first revisit the basic idea of multilevel error estimation and then apply this to our hierarchical setting. It will turn out that there are some modifications necessary when compared to standard finite element or T-spline refinement.

\subsubsection{Multilevel error estimation}

The main idea of multilevel error estimation is to enlarge the Galerkin subspace $\mathcal{V}_{h}$ by another, disjoint subspace $\mathcal{W}_{h} \subset \mathcal{V}$. This leads to a new subspace

$$
\widetilde{\mathcal{V}}_{h}=\mathcal{V}_{h} \oplus \mathcal{W}_{h}
$$

which is supposed to approximate the solution significantly better. Starting from the residual equation

$$
a(e, \psi)=\langle l, \psi\rangle-a\left(\varphi_{h}, \psi\right) \quad \text { for all } \psi \in \mathcal{V},
$$

we can state a weak formulation for an approximate error $e_{h} \in$ $\mathcal{W}_{h}\left(\right.$ instead of $\left.\widetilde{\mathcal{V}}_{h}\right)$ : given $\varphi_{h}$, find $e_{h} \in \mathcal{W}_{h}$ such that

$$
a\left(e_{h}, \psi\right)=\langle l, \psi\rangle-a\left(\varphi_{h}, \psi\right) \quad \text { for all } \psi \in \mathcal{W}_{h} .
$$

The error estimator $\eta$ is now defined for each element $T$ via

$$
\eta(T):=\left\|\left.e_{h}\right|_{T}\right\|
$$

This estimator is reliable and efficient if the saturation assumption

$$
\left\|\varphi-\widetilde{\varphi}_{h}\right\| \leq \gamma\left\|\varphi-\varphi_{h}\right\|
$$

with $\gamma<1$ independent of $h$ and the strict Cauchy inequality for $\left.a\right|_{\mathcal{V}_{h} \oplus \mathcal{W}_{h}}$ hold with fixed constants in the whole refinement algorithm (for further details see, e.g., [22]).

For a simple and efficient implementation, $\mathcal{W}_{h}$ is chosen as the function space spanned by the so-called bubble functions. The univariate bubble functions are defined with two real parameters $a<b$ as

$$
w[a, b](x)= \begin{cases}\frac{x-a}{b-a} \cdot \frac{b-x}{b-a}, & x \in(a, b) \\ 0, & \text { else. }\end{cases}
$$

Their support is restricted to the interval $[a, b]$, and the extension to two dimensions

$$
W_{k}(x, y)=w[a, b](x) \cdot w[c, d](y)
$$

has as support the quadrilateral element $[a, b] \times[c, d]$, which simplifies the evaluation of the estimator in (34).

\subsubsection{Error estimation for hierarchical isogeometric analysis}

A distinctive property of error estimators is their elementwise evaluation, which yields a spatial distribution of the error contributions. Using the set of active elements as introduced in Subsection 3.2.1, we can define the bubble functions (36) on their preimage and get as augmenting subspace

$$
\mathcal{W}_{h}=\operatorname{span}\left\{W_{k} \circ F^{-1}, k=1, \ldots, n_{e l}\right\}
$$

with $n_{e l}$ is the number of elements, i.e., $n_{e l}=|\mathcal{A}|$. This results in an estimation of the error on each element, and by means of a marking criterion such as (30), a set of elements to be refined can be selected.

However, this procedure has to be adapted to the hierarchical refinement as it is not always guaranteed that the refined grid does fulfill the support condition (23). One possibility to ensure this condition is a correction step after the elements have been marked, but there is another, easier way. The support condition demands that not only single elements but complete supports of the coarse basis functions are marked for refinement. A simple approach to ensure this is to transfer the element-wise error indicator to the basis functions by a modified error indicator $\tilde{\eta}: \Phi \rightarrow \mathbb{R}_{0}^{+}$that forms the average

$$
\tilde{\eta}(\varphi)=\frac{1}{|S(\varphi)|} \sum_{T \in S(\varphi)} \eta(T)
$$

with $S(\varphi):=\{T \in \mathcal{A}: T \subset \operatorname{supp} \varphi\}$. Analogously to the criterion (30), we mark the functions to be refined based on the data provided by $\tilde{\eta}$. In the mesh, only those elements that are in the union of the supports of the marked basis functions are refined. In this way the condition (23) is satisfied by construction.

The properties of this error estimation as well as other approaches are currently investigated.

\section{Computational examples}

In this section we present computational examples that demonstrate the hierarchical refinement algorithm. The selection of examples is inspired by [9] where T-spline refinement was investigated.

\subsection{Stationary heat conduction}

We start with the stationary heat conduction problem

$$
\Delta u=0
$$

on an L-shape domain $\Omega=[-1,1]^{2} \backslash[0,1]^{2}$, see Fig. 12, with boundary conditions such that the analytical solution is given by

$$
f(r, \varphi)=r^{\frac{2}{3}} \sin \left(\frac{2 \varphi-\pi}{3}\right)
$$




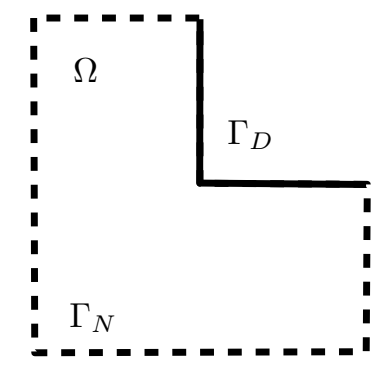

Figure 12: L-shape domain with Dirichlet boundary conditions on $\Gamma_{D}$ and Neumann boundary conditions on $\Gamma_{N}$

in polar coordinates $(r, \varphi)$.

A good error estimator is expected to detect the corner singularity and to trigger a local refinement in its neighborhood. A simulation with degree $p=q=2$ and parameter $\alpha=0.5$ in the marking criterion (30) yields the refined grid shown in Fig. 13. The adaptive isogeometric method performs well and generates the desired sequence of hierarchical meshes around the corner singularity. The refinement process for degree $p=q=3$ leads to very similar results.

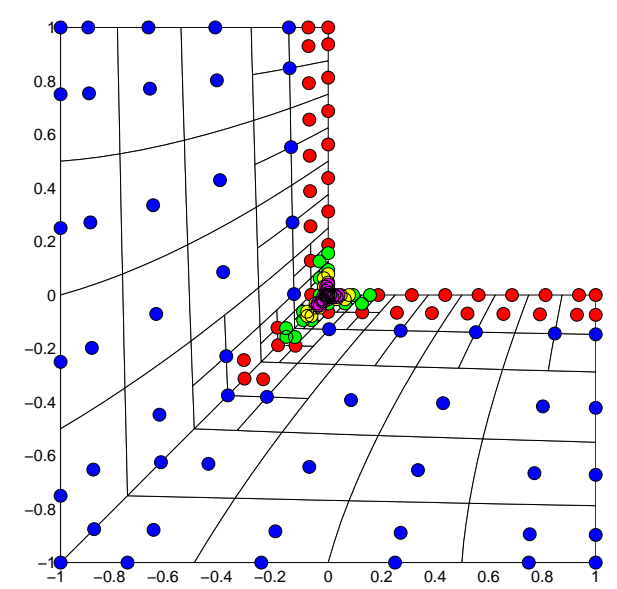

Figure 13: Refined grid on the L-shape for degree $p=q=2$

In order to obtain a more quantitative information about the error estimator we look at the ratio $\zeta:=\left\|e_{h}\right\| /\|e\|$ between estimated error $\left\|e_{h}\right\|$ computed by the estimator and the actual error $\|e\|$ for each refinement step. The results for the quadratic and cubic case are shown in Tab. 1. For both cases we see that the estimated error, although more pessimistic, remains within the order of magnitude of the actual error. Of course, this is just a basic numerical test and a complete analysis still has to be carried out and the theoretical properties need to be proven.

Finally, Fig. 14 contains convergence plots both for quadratic and cubic basis functions. With respect to the degrees of freedom (DOF) used to achieve a certain accuracy, the adaptive hierarchical approach is, as expected, superior to a uniform refinement.

Furthermore, due to the overlap of the basis function it is

\begin{tabular}{r|rrrr}
\multicolumn{2}{c}{ (a) $p=q=2$} & & \multicolumn{2}{c}{ (b) $p=q=3$} \\
dof & $\zeta$ & & dof & $\zeta$ \\
\hline 66 & 1.26 & & 91 & 2.05 \\
82 & 1.94 & & 165 & 1.58 \\
98 & 4.20 & & 192 & 3.02 \\
130 & 5.58 & & 219 & 5.63 \\
146 & 7.75 & & 288 & 8.12 \\
162 & 8.62 & & 315 & 10.10 \\
329 & 6.43 & & 486 & 7.87
\end{tabular}

Table 1: Ratio $\zeta$ between estimated and actual error for the L-shape problem

interesting to take a look at the condition of the resulting system. The condition number $k$ of the stiffness matrix for uniform as well as adaptive hierarchical refinement with degree 2 for the L-shape problem are listed in Tab. 2. On the one hand, we

\begin{tabular}{r|rrrrr}
\multicolumn{2}{c}{ (a) uniform } & & \multicolumn{2}{c}{ (b) adaptive } \\
dof & $\kappa$ & & dof & $\kappa$ \\
\cline { 5 - 6 } 66 & 67.7 & & 66 & 67.7 \\
190 & 231.7 & & 82 & 100.2 \\
630 & 847.4 & & 98 & 120.0 \\
2278 & 3235.5 & & 130 & 131.2 \\
8646 & 12652.9 & & 146 & 137.5
\end{tabular}

Table 2: Condition number $\kappa$ of the stiffness matrix of the L-shape problem

see the expected increase of $\kappa$ for the uniform refined case. In contrast, due to the comparably low degrees of freedom we still obtain moderate condition numbers in the adaptive case. Note that it was shown previously that the accuracy of the adaptively refined solution is higher than for the uniformly refined one. Nevertheless, due to the hierarchical nature of the refinement the ratio between condition number and degrees of freedom may be higher in the adaptive case. However, this issue depends on several points such as the underlying parameterization and requires further research.

\subsection{Elastic plate with circular hole}

The second example is the well-known infinite plate with a circular hole under in-plane tension in $x$-direction $[1,2]$. We study the stationary linear elasticity problem

$$
\operatorname{div} \sigma(u)=f
$$

under the assumption of plane stress. Due to symmetry the computational domain is restricted to a quarter. Furthermore we employ Dirichlet boundary conditions according to the analytical solution of the infinite plate at the boundary of our finite computational domain, Fig. 15.

This example is remarkable because by making use of NURBS, isogeometric methods are able to represent the given geometry exactly, including the circular hole. Hierarchical refinement inherits this feature, see the derivation of (27), and moreover the new weights of the refined NURBS are calculated by simple knot insertion. Starting from an initial parametrization of degree 2 with 28 DOF, we obtain the refined grid in Fig. 16. 


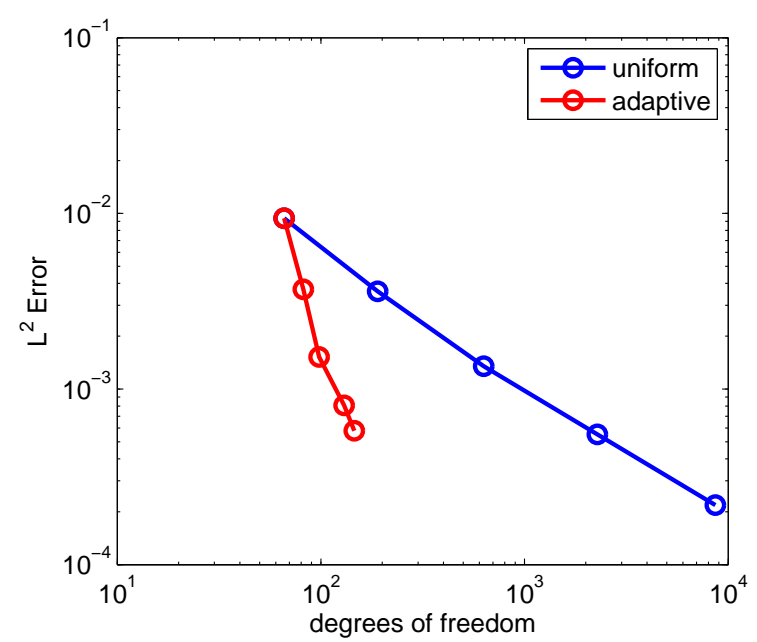

(a) degree $p=q=2$

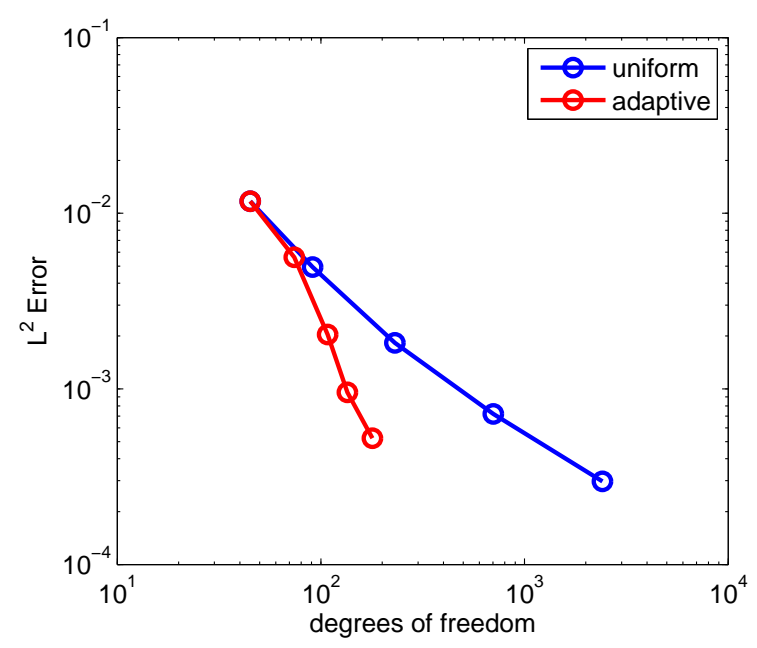

(b) degree $p=q=3$

Figure 14: Convergence plot for the L-shape problem

Fig. 17 displays the numerical solution, with colors indicating the stress distribution. Fig. 18 illustrates the convergence behavior of the first principal stress component at the point $(-\sqrt{2} / 2, \sqrt{2} / 2)$, which is located directly in the middle of the quarter circle with free boundary conditions. This region is primarily picked for refinement, and it can be seen that again the local refinement achieves similar precision with fewer DOF. The advantage over uniform refinement, however, is smaller than for the L-shape domain with its singularity.

\subsection{Advection-diffusion}

This benchmark example is also taken from [1, 2]. It consists of solving the advection-diffusion equation

$$
-\kappa \Delta u+v \cdot \nabla u=0
$$

on the unit-square with discontinuous Dirichlet boundary conditions (see Fig. 19). The diffusion coefficient is set to very small value $\left(\kappa=10^{-6}\right)$ compared to the advection velocity

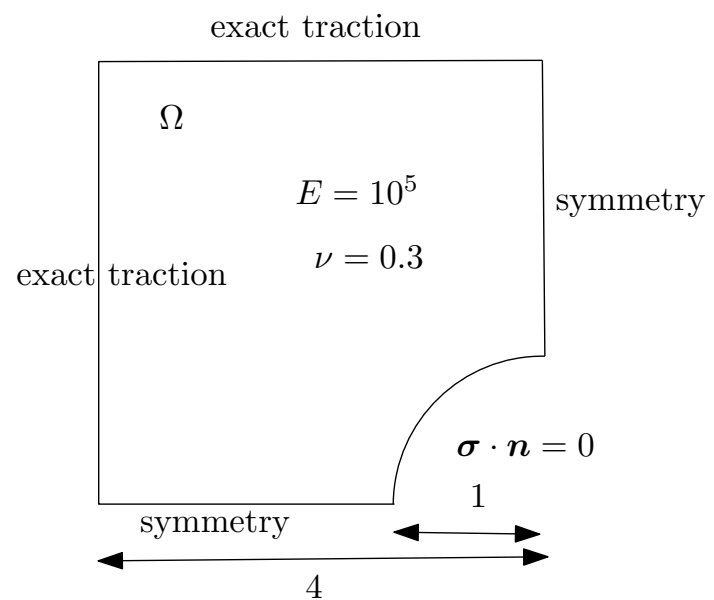

Figure 15: Elastic plate with circular hole

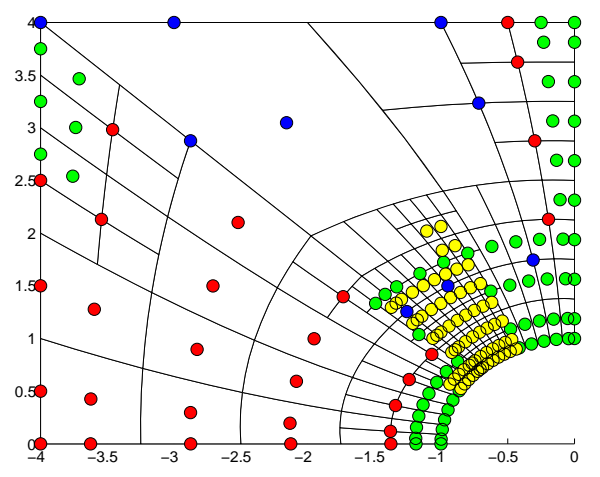

Figure 16: Grid after four refinement steps for the elastic plate with circular hole

$v=(\sin \theta, \cos \theta)^{T}$. Therefore, very sharp layers arise, and the equation needs to be stabilized in order to obtain reasonable numerical results. As in [1] and [9], we employ SUPG stabilization with the same parameter $\tau_{T}=h_{T} /(2\|v\|)$ where

$$
h_{T}=\operatorname{diam}(T) /(\sqrt{2} \max (\sin \theta, \cos \theta) .
$$

It turns out that the error estimator (39) introduced above is still not efficient for this challenging problem. To demonstrate the capabilities of hierarchical refinement, we use a heuristic strategy instead and mark at each level the elements that are affected by the sharp layers. Due to the diagonal expansion of the marked region, it is interesting how the refinement behaves and whether the refined elements spread over the domain or not.

Fig. 20 shows the refined grid for degree $p=q=3$. The refined region clearly stays near the initially marked selection. No undesirable propagation of grid points occurs as it may happen with T-splines [9]. The numerical solution, which is plotted in Fig. 21, resolves the sharp layers well due to the local refinement. 


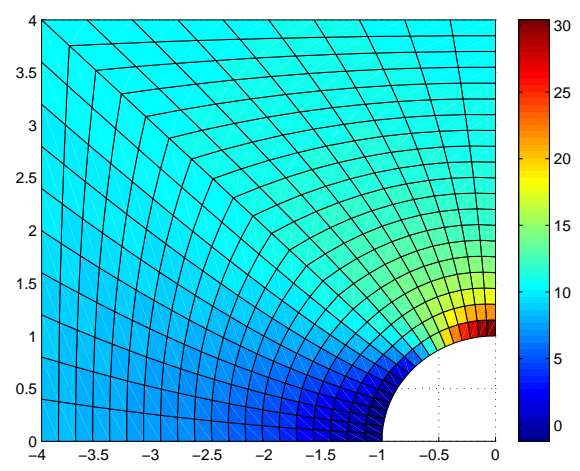

Figure 17: Numerical solution for plate with circular hole: first principal stress component

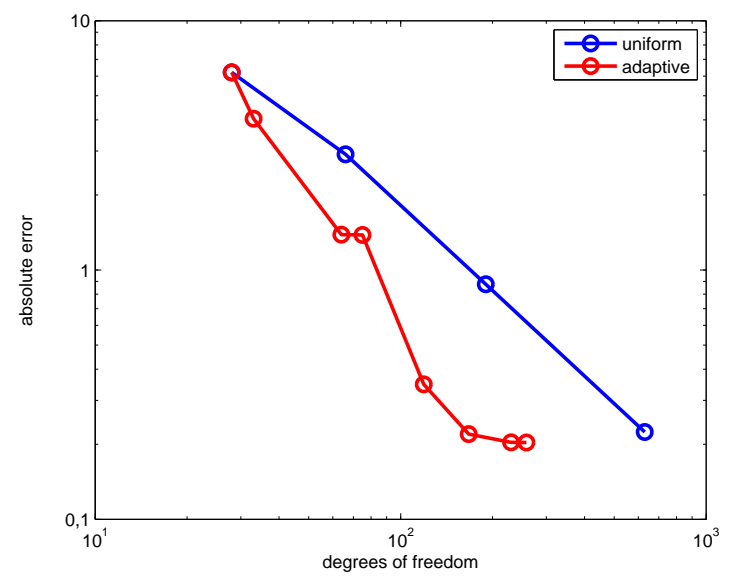

Figure 18: Pointwise convergence of the first principal stress component at $(-\sqrt{2} / 2, \sqrt{2} / 2)$

\section{Conclusions}

In this article we have discussed hierarchical B-splines as a locally refined spline space and how to use this concept for local refinement in isogeometric analysis. Hierarchical refinement turns out to be very flexible, because it is suitable for any degree or continuity in combination with B-splines or NURBS. Properties like linear independence, nested spaces and partition of unity can be assured right from the construction of the basis. Due to the simplicity of the concept, propagation of inserted knots does not occur which was also shown by some examples. Combined with an a posteriori error estimator the results of the numerical simulation of some test problems are very promising and further increase the efficiency notably.

Still, there are topics that will be the focus of future work. The idea of the described refinement technique works analogously in three dimensions and also coarsening is not more involved. An extension to multipatch problems is another possible topic requiring future research. Also only a special realization of the hierarchical concept was used for the simulations.

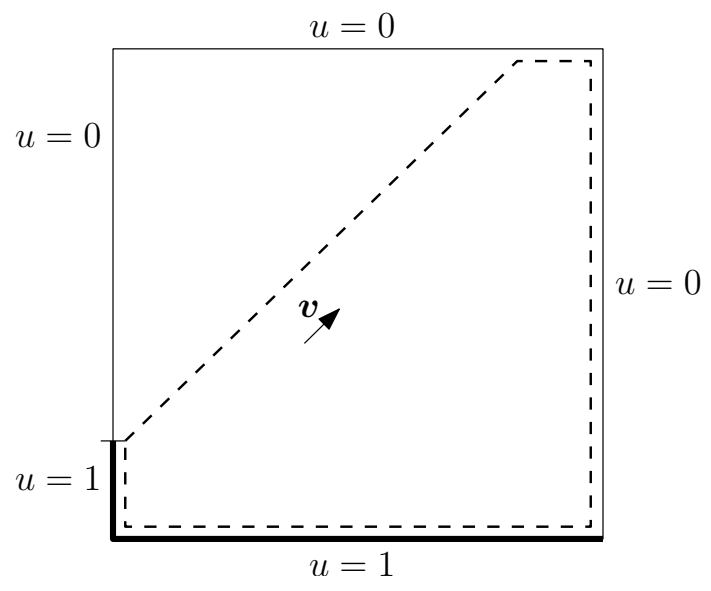

Figure 19: Advection-Diffusion problem

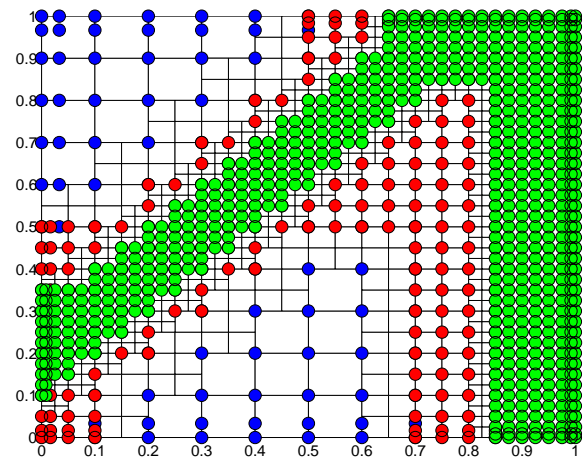

Figure 20: Refined grid for the advection-diffusion problem

Further adjustments and improvements include the use of less restrictive conditions on the refinement, which may lead to a more local behavior of the adaptive refinement process. An approach to increase the sparsity of the stiffness matrix (by using another basis of hierarchical splines) is currently under investigation; this may also improve the condition number. Error estimation in isogeometric analysis and especially combined with hierarchical refinement is just at an early stage and also topic of future research. Moreover, the extension of the refinement technique for e.g. including p-refinement or altering the supports of the basis functions are auspicious from the theoretical side as well as for simulations. Finally, the effect on the numerical linear algebra is a further open question.

\section{Acknowledgments}

The financial support by the European Union within the 7th Framework Programme, projects 218536 "EXCITING" and 272089 "PARADISE" is gratefully acknowledged. Furthermore, the authors thank the reviewers for their comments, which were very helpful for improving the manuscript. 


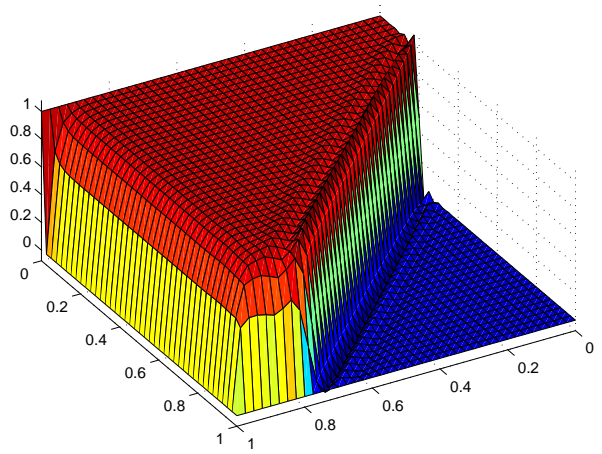

Figure 21: Numerical solution for the advection-diffusion problem

\section{References}

[1] T. J. R. Hughes, J. A. Cottrell, Y. Bazilevs, Isogeometric analysis: CAD, finite elements, NURBS, exact geometry and mesh refinement, Computer Methods in Applied Mechanics and Engineering 194 (2005) 4135-4195.

[2] J. A. Cottrell, T. J. R. Hughes, Y. Bazilevs, Isogeometric Analysis: Toward Integration of CAD and FEA, John Wiley \& Sons, 2009.

[3] Y. Bazilevs, V. M. Calo, T. J. R. Hughes, Y. Zhang, Isogeometric fluidstructure interaction: theory, algorithms, and compuations, Computational Mechanics 43 (2008) 3-37.

[4] W. A. Wall, M. A. Frenzel, C. Cyron, Isogeometric stuctural shape optimization, Computer Methods in Applied Mechanics and Engineering 197 (2008) 2976-2988.

[5] J. Kiendl, K.-U. Bletzinger, J. Linhard, J. Wüchner, Isogeometric shell analysis with Kirchhoff-Love elements, Computer Methods in Applied Mechanics and Engineering 198 (2009) 3902-3914.

[6] A. Buffa, G. Sangalli, R. Vázquez, Isogeometric analysis in electromagnetics: B-splines approximation, Computer Methods in Applied Mechanics and Engineering 199 (2010) 1143-1152.

[7] T. W. Sederberg, J. Zheng, A. Bakenov, A. Nasri, T-splines and TNURCCS, ACM Transactions on Graphics 22 (2003) 477-484.

[8] T. W. Sederberg, D. L. Cardon, G. T. Finnigan, N. S. North, J. Zheng, T. Lyche, T-spline simplification and local refinement, ACM Transactions on Graphics 23 (2004) 276 - 283.

[9] M. R. Dörfel, B. Jüttler, B. Simeon, Adaptive isogeometric analysis by local h-refinement with T-splines, Computer Methods in Applied Mechanics and Engineering 199 (2010) 264-275.

[10] Y. Bazilevs, V. M. Calo, J. A. Cottrell, J. Evans, T. J. R. Hughes, S. Lipton, M. A. Scott, T. W. Sederberg, Isogeometric analysis using T-Splines, Computer Methods in Applied Mechanics and Engineering 199 (2010) 229-263.

[11] A. Buffa, D. Cho, G. Sangalli, Linear independence of the T-spline blending functions associated with some particular T-meshes, Computer Methods in Applied Mechanics and Engineering 199 (2010) 1437-1445.

[12] X. Li, J. Zheng, T. W. Sederberg, T. J. R. Hughes, M. A. Scott, On linear independence of T-splines, ICES Report 10-40, University of Texas at Austin (2010).

[13] J. Deng, F. Chen, X. Li, C. Hu, T. W., Z. Yang, Y. Feng, Polynomial splines over hierarchical T-meshes, Graphical Models 70 (2008) 76-86.

[14] X. Li, J. Deng, F. Chen, Polynomial splines over general T-meshes, Visual Computer 26 (2010) 277-286.

[15] N. Nguyen-Thanh, H. Nguyen-Xuan, S. P. A. Bordas, T. Rabczuk, Isogeometric analysis using polynomial splines over hierarchical T-meshes for two-dimensional elastic solids, Computer Methods in Applied Mechanics and Engineering 200 (2011) 1892-1908.

[16] D. R. Forsey, R. H. Bartels, Hierarchical B-spline refinement, Computer Graphics 22 (1988) 205-212.

[17] R. Kraft, Adaptive and linearly independent multilevel B-splines, in: A. Le Méhauté, C. Rabut, L. L. Schumaker (Eds.), Surface Fitting and
Mulitresolution Methods, Vanderbilt University Press, Nashville, 1997, pp. 209-218.

[18] B. Wohlmuth, Discretization methods and iterative solvers based on domain decomposition, volume 17 of Lecture Notes in Computational Science and Engineering, Springer, 2001.

[19] P. Deuflhard, P. Leinen, H. Yserentant, Concepts of an adaptive hierarchical finite element code, IMPACT Comput. Sci. Eng. 1 (1989) 3-35.

[20] T. J. R. Hughes, The Finite Element Method, Dover Publ., Mineola, New York, 2000.

[21] Y. Bazilevs, L. B. Beirão da Veiga, J. A. Cottrell, T. J. R. Hughes, G. Sangalli, Isogeometric analysis: Approximation, stability and error estimates for h-refined meshes, Mathematical Methods and Models in Applied Sciences 16 (2006) 1031-1090.

[22] R. E. Bank, R. Smith, A posteriori error estimates based on hierarchical bases, SIAM Journal Numerical Analysis 30 (1993) 921-935. 